\title{
The use of infrared thermal imaging to measure spatial and temporal sweat retention in clothing
}

\author{
Margherita Raccuglia $^{1} \cdot$ Christian Heyde ${ }^{2} \cdot$ Alex Lloyd $^{1} \cdot$ Simon Hodder $^{1} \cdot$ George Havenith $^{1}$
}

Received: 12 June 2018 /Revised: 16 November 2018 / Accepted: 22 February 2019 /Published online: 27 March 2019

(C) The Author(s) 2019

\begin{abstract}
In our previous laboratory study a 'destructive' gravimetric method was developed to quantify local garment sweat absorption. While this currently is the only methodology that permits direct and analytical measurements of garment regional sweat absorption, the latter approach is time-consuming and expensive, therefore, of limited applicability. As such, in this study, we wanted to assess whether infrared thermography could be used as an indirect method to estimate garment regional sweat absorption, right after exercise, in a 'non-destructive' fashion. Spatial and temporal sweat absorption data, obtained in our previous study, were correlated with spatial and temporal temperature data obtained in the same experiment with an infrared thermal camera. The data suggest that infrared thermography is a good tool to qualitatively predict regional sweat absorption in garments at separate individual time points; however, temporal changes are not predicted well, due to a moisture content threshold above which variations in sweat content cannot be discriminated by further temperature changes.
\end{abstract}

Keywords Infrared thermography $\cdot$ Clothing sweat absorption $\cdot$ Clothing sweat mapping $\cdot$ Clothing evaporative cooling

\section{Introduction}

Temperature and moisture management in clothing is a main focus of the clothing industry with regard to garment performance optimisation and wear discomfort reduction. Liquid moisture content and transfer properties of fabrics can be assessed with a range of material test methods. In these tests, physical wetness of fabrics is induced by the investigator and/ or specific apparatus, by adding water or special solutions. Moisture properties of fabrics are then measured via the application of different technologies, e.g. gravimetric, observation, optical, electrical and temperature-based methods (Tang et al. 2014b). Although these tests are quick, easy and relatively cost-effective, they do not fully simulate the conditions in which liquid moisture absorption and transfer occur, such as in the clothed human body during physical work. In real-life use, immediately after physical exercise, a gravimetric

George Havenith

G.Havenith@lboro.ac.uk

1 Environmental Ergonomics Research Centre, Loughborough Design School, Loughborough University,

Loughborough, Leicestershire LE11 3TU, UK

2 Adidas FUTURE Sport Science, Herzogenaurach, Germany method, based on weight changes (difference between wet and dry garment weight), is typically adopted to estimate sweat absorption in a full garment (Baker et al. 2017). Nevertheless, to the knowledge of the authors, there are no standardised test methods able to directly measure liquid moisture content in specific clothing sections, without dissecting the garment. In our previous study (Raccuglia et al. 2017), a 'destructive' gravimetric method was developed to quantify garment regional sweat absorption. In this test, each T-shirt was cut into sections immediately following exercise, and based on weight changes of each section, local sweat content of each region of interest (ROI) of the garment was then determined. While this currently is the only methodology that permits direct and analytical measurements of garment regional sweat absorption, especially when studying temporal changes, the latter approach is time-consuming and expensive, therefore, of limited applicability.

In the building industry, infrared thermography (IRT) is used as diagnostic tool to detect the presence of damp in the cavity of walls and floors or the deterioration of historic structures due to moisture infiltration (Balaras and Argiriou 2002; Avdelidis et al. 2003). IRT involves the use of an infrared camera which can detect thermal radiation and produce colour images, termed as thermograms (Ring and Ammer 2000). A thermogram contains temperature data, and one of the main 
advantages is that it allows us to visualise temperature differences across the object captured, using colour differences that are related to a colour-temperature scale. Taking a cue from the building industry, given that textiles also cool when water (sweat) is present, we wanted to assess whether IRT could be applied to detect liquid moisture content in clothing and, more importantly, quantify spatial variation in this liquid content across the garment.

The improved sensitivity of infrared cameras (approximately $0.05^{\circ} \mathrm{C}$ ) allows detections of small temperature differences across objects examined, which is of crucial importance when adopting a temperature mapping approach (Fournet 2013; Fournet et al. 2013; Gerrett et al. 2015). The method is non-invasive and non-destructive, and does not require contact with the object examined (Formenti et al. 2016). The acquisition of the infrared images is quick and easy to perform; however, various protocols, guidelines and checklist must be followed (IACT 2002; ISO 9886:2004; Ammer 2008; Mercer and Ring 2009; Moreira et al. 2017) in order to prevent bias and obtain good quality data. For instance, attention should be paid to the position of the camera, distance of the camera from the object captured, operating and object temperature ranges as well as additional sources of calibration (mainly due to the absolute low accuracy, $\pm 2{ }^{\circ} \mathrm{C}$ ).

As a result of the numerous advantages, IRT has been used for a number of different applications (Moreira et al. 2017), including sport-related injuries prevention and treatment (Hadžić et al. 2015), activation of brown adipose tissue in the body (Robinson et al. 2016), assessments of cryotherapy protocols (Costello et al. 2012; Selfe et al. 2014; Silva et al. 2017) and measurements of skin temperature following aerobic and resistance exercise (Ferreira et al. 2008; Priego Quesada et al. 2015). Furthermore, IRT has been applied as tool to gain original insight regarding skin temperature patterns across the body in exercise and during cold (Fournet 2013; Fournet et al. 2013) and hot (Gerrett et al. 2015) exposure. This information can find application in clothing development using a body mapping approach, e.g. with spatial variations in textile type. Despite the wide range of applications, it has not been reported whether IRT can be applied to quantify sweat retention in clothing, following physical exercise. When the garment is on the body, its temperature is the result of dry heat loss to the environment, evaporative heat loss from the wet areas and heat input from the skin. However, when taken off the body, garment areas presenting higher sweat content would be affected by higher local (evaporative) cooling, resulting in higher temperature drop (from dry); therefore, a relationship between local sweat/ water content and local temperature drop was expected. As such, in this study, spatial and temporal sweat absorption data (obtained from our previous study) were correlated with spatial and temporal temperature data, measured using infrared thermography. Taking into account the influence of body skin temperature on local garment temperature, which could affect the hypothesised relationship between local sweat content and temperature, in the current study, infrared image acquisition was performed after removal of the T-shirt from the body.

Acknowledging the lack of time- and cost-effective test methods, the ultimate goal of this study was to assess whether IRT could be used as an indirect method to estimate garment regional sweat content in a quick and 'non-destructive' fashion. Furthermore, the fast image acquisition could allow assessments of garment sweat content immediately after physical exercising, minimising the risk for moisture migration and moisture evaporation from the garment, which is the main drawback of some lengthy tests (Tang et al. 2014b).

\section{Material and methods}

The infrared images of the garments were collected in parallel with the data collection conducted for our previous study (Raccuglia et al. 2017). Therefore, participants, exercise protocol and conditions were the same as those in this reference. The latter are described below.

\section{Participants}

Eight male, long distance runners were recruited from the Loughborough University student cohort. Participants' characteristics (mean and standard deviation) are reported in Table 1 . The experimental procedures where fully explained to the participants verbally and through written information form, before obtaining written informed consent and completing a health screening questionnaire. All the experimental procedures involved were approved by the Loughborough University Ethical Committee. The study was conducted within the confines of the World Medical Association Declaration of Helsinki for medical research involving human participants.

\section{Experimental conditions}

Sweat absorption and temperature across the T-shirt were mapped over a total running time of $50 \mathrm{~min}$ (Raccuglia et al. 2017). As a destructive gravimetric method was adopted to quantify regional sweat absorption, each participant performed 10 separate running trials on a treadmill, characterised by different durations: $5 \mathrm{~min}, 10 \mathrm{~min}, 15 \mathrm{~min}, 20 \mathrm{~min}, 25 \mathrm{~min}$, $30 \mathrm{~min}, 35 \mathrm{~min}, 40 \mathrm{~min}, 45 \mathrm{~min}$ and $50 \mathrm{~min}$. The 10 running trials were performed on different days, separated by at least $24 \mathrm{~h}$ of rest. Including the pre-test, each participant performed 11 visits in total and undertook only one running trial per day. Immediately after each partial running trial, the T-shirt was removed from the body and two infrared images of the T-shirt, one for the front and one for the back, were taken for each 
Table 1 Participants' characteristics

\begin{tabular}{lccccccc}
\hline & $\begin{array}{l}\text { Age } \\
(\text { years })\end{array}$ & $\begin{array}{l}\text { Weight } \\
(\mathrm{kg})\end{array}$ & $\begin{array}{l}\text { Height } \\
(\mathrm{cm})\end{array}$ & $\begin{array}{l}\mathrm{BSA} \\
\left(\mathrm{cm}^{-2}\right)\end{array}$ & $\begin{array}{l}\text { Body fat } \\
(\%)\end{array}$ & $\begin{array}{l}\mathrm{VO}_{2 \max } \\
\left(\mathrm{mL} \mathrm{kg}^{-1} \mathrm{~min}^{-1}\right)\end{array}$ & $\begin{array}{l}\text { Running speed } \\
\left(\mathrm{km} \mathrm{h}^{-1}\right)\end{array}$ \\
\hline Mean & 23.3 & 70.0 & 177.3 & 1.9 & 9.6 & 62.1 & 12.1 \\
STDEV & 4.7 & 9.9 & 5.3 & 0.1 & 4.5 & 3.1 & 0.7 \\
\hline
\end{tabular}

$B S A$ body surface area, $V O_{2 \max }$ maximum oxygen uptake, STDEV standard deviation participant. Therefore, for each person, with 10 trials and 2 pictures per trial, 20 thermograms were taken: 160 in total. A procedure similar to that developed by Fournet (2013; see image aquisiton and image processing sections) was adopted to obtained quantitative temperature data of the ROI and to provide average thermal patterns (temperature distribution across the garment), visually accessible with a colour scale. After taking the infrared picture, the T-shirt was dissected into 22 different regions of interest (ROI), presented in Fig. 1. Using a gravimetric approach (wet weight-dry weight), the time-course and distribution of sweat absorption of each garment were defined (Raccuglia et al. 2017). The experiment was conducted in a climate-controlled chamber maintained at $27.2 \pm 0.2{ }^{\circ} \mathrm{C}, 49.7 \pm 3.2 \%$ relative humidity $(\mathrm{Rh})$ and $1.5 \mathrm{~m} \mathrm{~s}^{-1}$ wind speed.

\section{Experimental garment}

A short sleeved, $100 \%$ cotton T-shirt was used for each of the 10 run durations. The T-shirt presented a regular fit and a surface area of approximately $0.8 \mathrm{~m}^{2}$. Specifications of the experimental garment are reported in Table 2.

\section{Infrared thermal camera}

A FLIR T620 (FLIR Systems Inc. Wilsonville, USA) infrared camera with 13.1-mm lens was used. The camera has an operating temperature range between -15 and $+50^{\circ} \mathrm{C}$, and an object temperature range between -40 and $+150{ }^{\circ} \mathrm{C}$, which encompasses the temperature range we aimed to examine (20$30^{\circ} \mathrm{C}$ ). The camera has a $640 \times 480$ pixel infrared resolution and the spectral range is $7.5-14 \mu \mathrm{m}$. Emissivity was set at 0.95 . The accuracy of the camera is of $\pm 2{ }^{\circ} \mathrm{C}$, which is low compared to the accuracy of other commonly used contact methods ( $\pm 0.5- \pm 0.1)$ (Fournet 2013). For this reason, a black body calibrator was included in the pictures to overcome this limitation (Fig. 2). This improved the accuracy to $0.5^{\circ} \mathrm{C}$ and had a stability of $0.1{ }^{\circ} \mathrm{C}$. Despite the poor intrinsic accuracy, the camera presents a very high thermal sensitivity of \pm $0.04{ }^{\circ} \mathrm{C}$. The high thermal sensitivity allowed detection of very small spatial and temporal changes in the temperature of the garment, which is crucial in a temperature mapping approach. Given the high stability of the IR calibrator and the camera itself, combined with the high sensitivity of the camera, the relative measurements required had sufficient accuracy for the present application. In accordance with

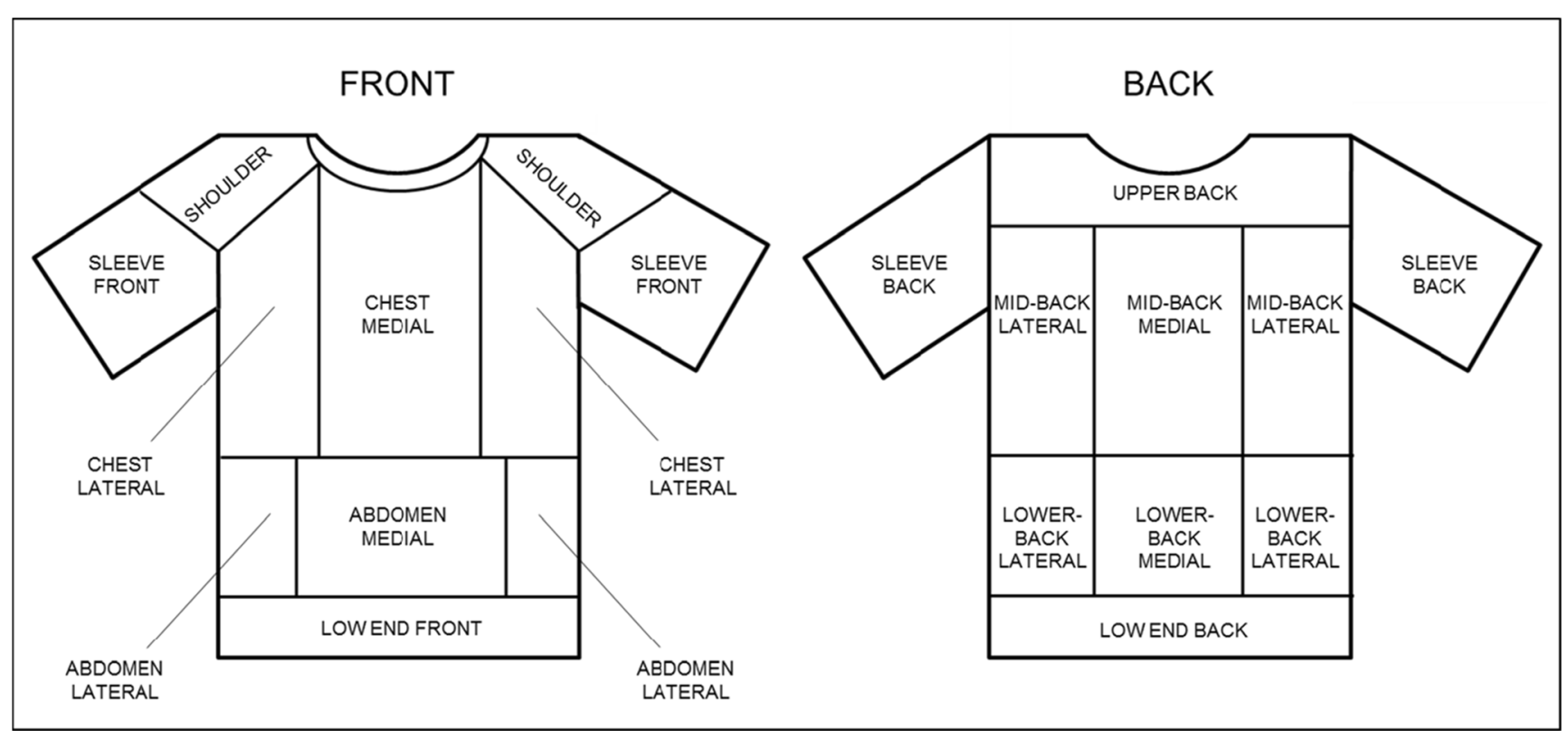

Fig. 1 Schematic representation of the experimental T-shirt marked into the 21 regions of interest for the analyses of local sweat accumulation. Front and back of the T-shirt were mapped into 11 and 10 zones, respectively 
Table 2 Specifications of the experimental garments

\begin{tabular}{lllllll}
\hline $\begin{array}{l}\text { Fibre } \\
\text { Content }\end{array}$ & $\begin{array}{l}\text { Mass } \\
\left(\mathrm{g} \mathrm{m}^{-2}\right)\end{array}$ & $\begin{array}{l}\text { Thickness } \\
(\mathrm{mm})\end{array}$ & $\begin{array}{l}\mathrm{R}_{\mathrm{ct}} \\
\left(\mathrm{m}^{2}{ }^{\circ} \mathrm{C} / \mathrm{W}\right)\end{array}$ & $\begin{array}{l}\mathrm{R}_{\mathrm{et}} \\
\left(\mathrm{m}^{2} \mathrm{~Pa} / \mathrm{W}\right)\end{array}$ & $\begin{array}{l}\text { Air perm } \\
\left(\mathrm{mm} \mathrm{s}^{-1}\right)\end{array}$ & $\begin{array}{l}\text { Absorption } \\
\left(\mathrm{g} \mathrm{m}^{-2}\right)\end{array}$ \\
\hline $100 \%$ cotton & 159 & 0.55 & 0.02 & 3.1 & 780 & 381 \\
\hline
\end{tabular}

$\mathrm{R}_{\mathrm{ct}}=$ dry thermal resistance; $\mathrm{R}_{\mathrm{et}}=$ water vapour resistance, Air perm = air permeability, Absorption $=$ total absorption capacity. Dry thermal resistance and water vapour resistance were measured according to BS EN ISO 11092:2014, air permeability was measured according to BS EN ISO 9237:1995; total absorption capacity was measured according to the absorption capacity test adopted by Raccuglia et al.(2016), modified from Tang et al. (2014a) guidelines (Moreira et al. 2017), the camera was switched on in advance to allow stabilisation.

As apparent temperature differences in thermograms can arise from potential curvatures when the obliquity is larger than $45^{\circ}$ (Watmough et al. 1970), it is important to point out that the quality of the measurement was not affected by geometry-related issues, due to the flat shape of the garment.

\section{Image acquisition}

A standardised procedure was developed for the acquisition of the infrared images. At the end of each running trial, the wet garment was removed from the body and fitted to a custommade T-shirt-like shape wooden stand (Fig. 2), which was treated with a hydrophobic finish to prevent water transfer from the T-shirt to the stand. Image acquisition occurred always 3 min after taking the T-shirt off the body to allow the textile to cool. The stand was positioned at a fixed location,

\section{FRONT}
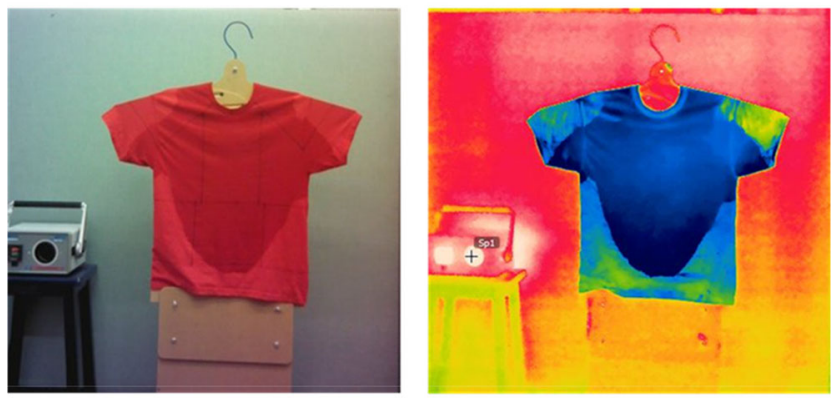

BACK
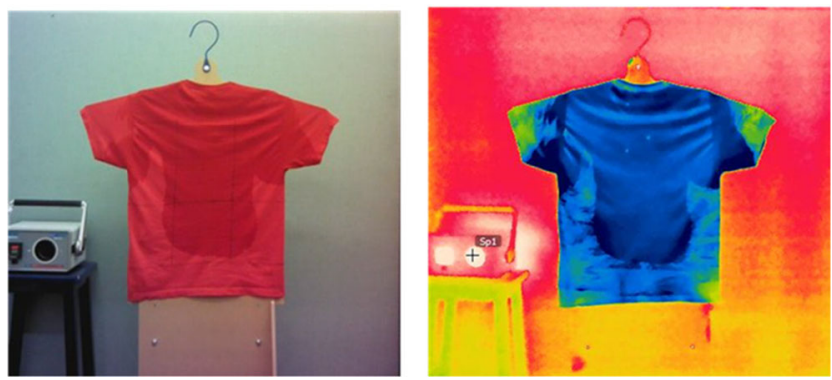

Fig. 2 Digital and Infrared pictures of identical views for front and back of the T-shirt, including black body calibrator positioned on a stool behind the T-shirt stand and the camera was fitted to a tripod, at a distance of $2 \mathrm{~m}$ perpendicular to the T-shirt stand. The black body calibrator was included in the background of each thermogram (Fig. 2) so that potential measurement errors could be reduced.

\section{Image processing}

Image processing was performed with three main goals (Fournet 2013). The procedure allowed (1) standardising the analysis of the numerous thermograms, (2) segmentation of each thermogram into the 21 ROIs as well as extracting the important spatial temperature data of each ROI, and (3) creating average thermograms of the T-shirt at each time point (trials, from 5- to 50-min run duration).

The development of the image processing procedure was performed using the software MATLAB 7.8.0 (MATLAB R2013a, The MathWorks Inc., Natick, USA). MATLAB scripts modified from those developed by Fournet (2013) were used for the analysis. The image processing involved morphing, averaging and creation of average maps for each trial (time point). Specifically, to account for differences in Tshirt size and position (although it was standardised as much as possible with the T-shirt stand), all thermograms were morphed (i.e. adapted) onto a single reference T-shirt shape, now having the exact same shape, size and position in the images. Following from this, the individual morphed thermograms were averaged to obtain a final single T-shirt map of temperature distribution, for each running duration. Figure 3 summarises the different stages of the image processing procedure. Furthermore, after being morphed, each thermogram was segmented into the 21 ROIs, according to Fig. 1, and regional temperature data $\left(\right.$ Temp $\left._{\text {Local }}\right)$ were computed to calculate the average local temperatures.

Due to the evaporative cooling provided by the presence of liquid sweat, we hypothesised that garment regions with greater sweat content will result in lower temperature as compared to the temperature of the T-shirt in dry state or lower sweat content. As in dry state, the temperature of the T-shirt by definition equals ambient temperature, and the temperature of each garment region was considered as temperature drop from ambient temperature, according to: 
Fig. 3 Image processing sequence using MATLAB. Each individual thermogram is morphed to the reference thermogram. The individual thermograms are then averaged for the creation of a final T-shirt map of temperature distribution

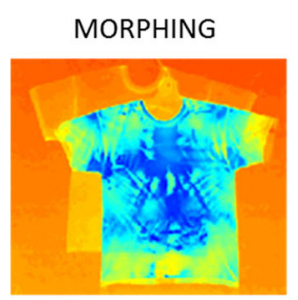

AVERAGING

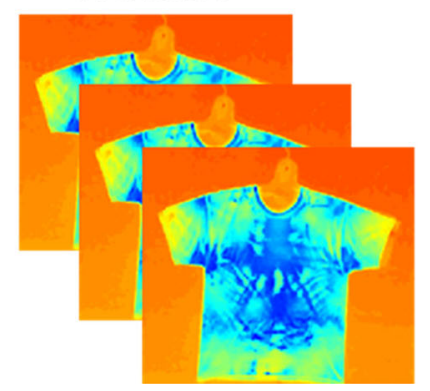

FINAL MAP

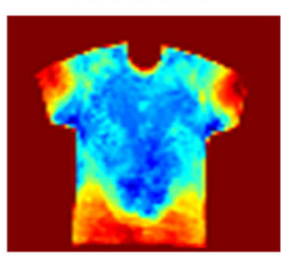

TempDrop $_{\text {Local }}\left({ }^{\circ} \mathrm{C}\right)=T_{\text {ambient }}-$ Temp $_{\text {Local }}$

where

Temp $_{\text {Local }}$ garment local absolute temperature in ${ }^{\circ} \mathrm{C}$

$T_{\text {ambient }}$ temperature of the ambient air in ${ }^{\circ} \mathrm{C}$

\section{Statistics}

Regression analyses were performed to study the relation between garment local sweat absorption $\left(\mathrm{ABS}_{\text {Local }}\right)$ and temperature data (Temp-Drop Local $_{\text {) }}$. The regression analyses were performed using different data sub-sets. One regression model included participants' individual data for each ROI at all running durations ( 8 participants $\times 21$ ROIs $\times 10$ running durations). Another model included participants' average data of the ROIs at all running durations ( 21 averaged ROIs $\times 10$ running durations). Temporal models, including participants' average data of the ROIs, were provided separately for four selected individual running durations ( $15 \mathrm{~min}, 25 \mathrm{~min}, 35 \mathrm{~min}$ and $50 \mathrm{~min}$ ) (21 averaged ROIs $\times 1$ running duration).

$\mathrm{ABS}_{\text {Local }}$ was plotted versus Temp-Drop Local $_{\text {at }}$ each running duration (5-50 min) for single selected ROI (chest medial, back upper, shoulder, back mid lateral, shoulder, abdomen medial, low end back) (10 running durations $\times 1$ averaged ROI), and descriptive statistics were performed.

The assumption of normality of distribution of the residuals was checked with histograms and Normal P-P plots. The assumption of homoscedasticity of the residuals was checked using scatter plots of the studentised residuals against unstandardised predicted values. When these assumptions were violated, data transformations were conducted.

To characterise the strength of the relations, coefficient of determination $\left(r^{2}\right)$ as well as standard errors of the estimate (SEE) were calculated. Data were analysed using the software IBM SPSS Statistics (version 22) (IBM, USA).

\section{Results}

Temperature and sweat maps of front and back side of the garments are illustrated in Fig. 4a, b.

\section{Regression models}

$\mathrm{ABS}_{\text {Local }}$ ranged from 0 to $293 \mathrm{~g} \mathrm{~m}^{-2}$ and TempDrop $_{\text {Local }}$ ranged from 0 (no drop) to $4.3{ }^{\circ} \mathrm{C}$.

Whilst an exponential function was found to best describe the data (Fig. 5a), the assumptions of normality of distribution and homoscedasticity of the residuals were violated; hence, data transformation was required.

Data transformation included an exponential transformation of Temp-Drop Local $\left(e^{\text {TempDropLocal }}\right)$ and a logarithmic transformation of $e^{\text {TempDropLocal }}\left({ }^{10} \log \left(e^{\text {TempDropLocal }}\right)\right)$ and $\mathrm{ABS}_{\text {Local }}\left({ }^{10} \log \mathrm{ABS}_{\text {Local }}\right)$. After performing data transformation, the residuals displayed a normal distribution and homoscedasticity (Fig. 5b). The predictive power of the regression model was statistically significant $(p<0.001)$, but the coefficient of determination was relatively low $\left(R^{2}=0.52\right)$ (Table 3, overall individual model).

The assumptions of normality of distribution and homoscedasticity of the residuals were also violated in the overall average model (21 ROIs averaged over participants $\times 10$ running durations). As such, the same transformations were applied to the two variables, considered as ${ }^{10} \log \left(e^{\text {TempDropLocal }}\right)$ and ${ }^{10} \mathrm{Log} \mathrm{ABS}_{\text {Local }}$. The overall average model presented higher coefficient of determination $\left(R^{2}=0.75\right)$, compared to the individual model and also reached statistical significance $(p<0.001)$ (Table 3, overall average). The predictive equations of the individual and average overall models are reported in Table 4.

Exponential curves were found to best fit the relation between $\mathrm{ABS}_{\text {Local }}$ and TempDrop Local $_{\text {, at each selected running }}$ duration, i.e. $15 \mathrm{~min}, 25 \mathrm{~min}, 35 \mathrm{~min}$ and $50 \mathrm{~min}$ (Fig. 6), with curves shifting up and left with advancing time. The temporal models were statistically significant $(p<0.001)$, and their predictive power $\left(R^{2} \geq 0.75\right)$ was higher than the overall models (Table 3 ). The predictive equations of the temporal models are reported in Table 4. 


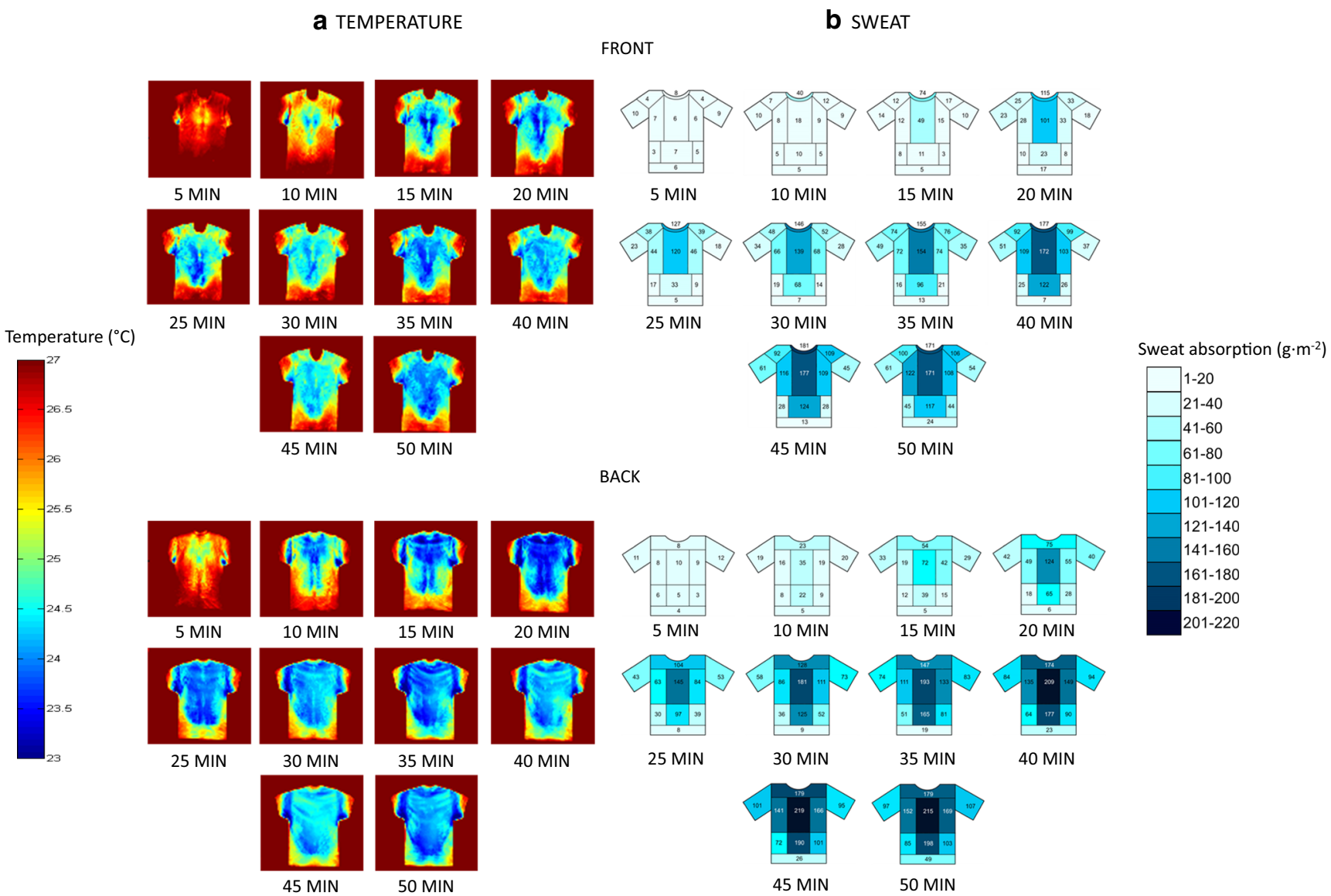

Fig. 4 Average (eight participants) maps of temperature (panel $\mathbf{a}$, left) and sweat (panel $\mathbf{b}$, right) distribution across the front side of the garments, over 50 min of running exercise. Data for each time point were obtained from 10 different running trials

\section{Sweat absorption and temperature in single ROI}

In each selected ROI (10 running duration $\times 1$ averaged ROI),

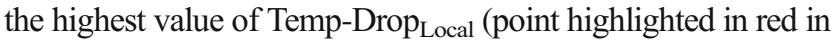
Fig. 7) occurred before $\mathrm{ABS}_{\text {Local }}$ could reach its highest value. Specifically, the highest TempDrop Local was $2.79 \pm 0.53^{\circ} \mathrm{C}$ for chest medial, $3.24 \pm 0.35{ }^{\circ} \mathrm{C}$ for back upper, $2.78 \pm 0.70{ }^{\circ} \mathrm{C}$ for back mid lateral, $2.47 \pm 0.67$ for shoulders, $1.89 \pm 0.69$ for abdomen medial, and $2.08 \pm 0.85{ }^{\circ} \mathrm{C}$ for low end back. Highest

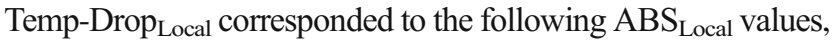

$49.1 \pm 40.1 \mathrm{~g} \mathrm{~m}^{-2}$ for chest medial, $74.8 \pm 27.2 \mathrm{~g} \mathrm{~m}^{-2}$ for back upper, $55.13 \pm 30.23 \mathrm{~g} \mathrm{~m}^{-2}$ for back mid lateral, $47.9 \pm$ $29.2 \mathrm{~g} \mathrm{~m}^{-2}$ shoulder, $33.3 \pm 21.5 \mathrm{~g} \mathrm{~m}^{-2}$ for abdomen medial, and $49.2 \pm 31.1 \mathrm{~g} \mathrm{~m}^{-2}$ for low end back.

\section{Discussion}

The main focus of this investigation was to determine whether infrared thermography can be used to quantify moisture in
Fig. 5 a Exponential relationship between local sweat absorption $\left(\mathrm{ABS}_{\text {Local }}\right)$ and local temperature drop (TempDrop Local ). The model includes participants' individual data for each ROI at each running duration ( 8 participants $\times 21$ ROIs $\times 10$ running durations). $\mathbf{b}$ Plots of Studentised residuals against unstandardised predicted values, after data transformation a

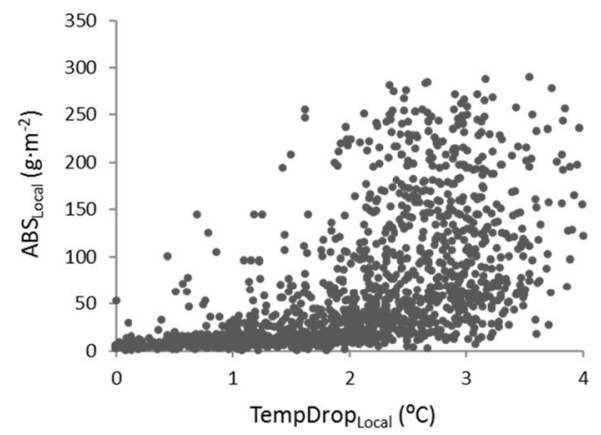

b

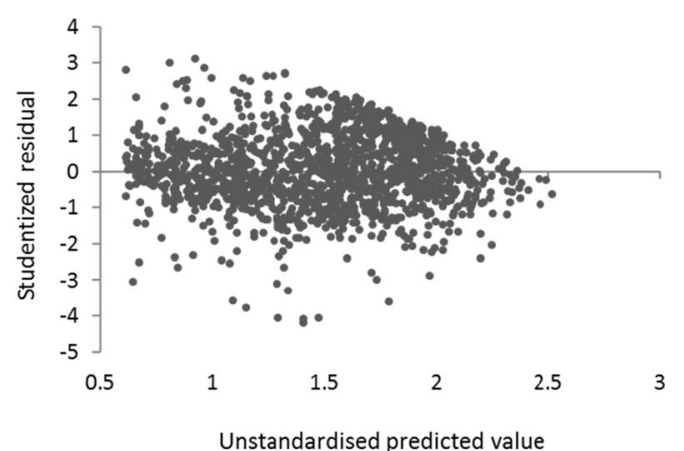


Table 3 Summary of the overall (individual and average) and temporal model and variables included

\begin{tabular}{|c|c|c|c|c|c|c|}
\hline \multicolumn{2}{|l|}{ Model } & Predicted variable & Independent variable & $R^{2}$ & SEE & Sig. \\
\hline \multicolumn{2}{|c|}{ Overall individual } & ${ }^{10} \log \left(\mathrm{ABS}_{\text {Local }}\right)$ & ${ }^{10} \log \left(e^{\text {TempDroplocal }}\right)$ & 0.52 & 0.40 & $<0.001$ \\
\hline \multicolumn{2}{|c|}{ Overall average } & ${ }^{10} \log \left(\mathrm{ABS}_{\text {Local }}\right)$ & ${ }^{10} \log \left(e^{\text {TempDroplocal }}\right)$ & 0.75 & 0.25 & $<0.001$ \\
\hline \multirow[t]{4}{*}{ Temporal } & $15 \mathrm{~min}$ & $\mathrm{ABS}_{\text {Local }}$ & $e^{\text {TempDroplocal }}$ & 0.75 & 8.12 & $<0.001$ \\
\hline & $26 \min$ & $\mathrm{ABS}_{\text {Local }}$ & $e^{\text {TempDroplocal }}$ & 0.87 & 11.67 & $<0.001$ \\
\hline & $35 \mathrm{~min}$ & $\mathrm{ABS}_{\text {Local }}$ & $e^{\text {TempDroplocal }}$ & 0.82 & 28.63 & $<0.001$ \\
\hline & $50 \mathrm{~min}$ & $\mathrm{ABS}_{\text {Local }}$ & $e^{\text {TempDroplocal }}$ & 0.88 & 21.81 & $<0.001$ \\
\hline
\end{tabular}

SEE standard error of estimate garments, by developing a relation between the amount of moisture absorbed and the temperature drop of wet textile areas after taking off the garment. Whilst IRT has been adopted to provide maps of temperature distribution across the human body (Fournet 2013; Fournet et al. 2013), so far, IRT has not been used as tool to predict spatial and temporal sweat retention in clothing, after physical exercise. The data suggest that IRT is a good tool to qualitatively predict regional sweat absorption in garments at separate individual time points; however, temporal changes are not predicted well, likely due to a moisture threshold above which variations in sweat content do not lead to further temperature changes and thus cannot be discriminated by further temperature changes.

\section{Overall models}

We assumed that garment zones characterised by higher sweat retention would be affected by higher evaporative cooling, resulting in higher local temperature drops from their dry state. This was the rationale for attempting to use temperature data to estimate sweat content in clothing. In order to ensure a direct link between garment regional sweat retention and temperature, infrared pictures of the garment were performed after taking the garment off the body. In fact, pilot testing for this study showed that acquisition of the picture while the garment was still on the body gives a combined value of garment and body skin temperature. As such, when the purpose is to assess garment regional sweat content using infrared temperature data, it is important to remove the garment from the body, fit it to a garment-like shape stand and wait for about $3 \mathrm{~min}$ to stabilise. The stand also allows separation of the front from the back-T-shirt's panel (preventing sweat transfer between front and back regions) as well as avoiding infrared transmission from the other side through the garment and ensures a standardised T-shirt position.

While the overall model including spatial and temporal data of the eight participants was highly significant (individual model, Table 3), regional temperature drop only statistically explained $52 \%$ of the variance in regional sweat content (Table 3). This suggests that other factors might have affected the link between these two parameters. The exponential shape of the curves describing the relation between local sweat absorption and temperature indicates that local temperature changes can predict local sweat retention up to a certain moisture saturation value, this being around $50 \mathrm{~g} \mathrm{~m}^{-2}$ (in a cotton material and in the climatic condition adopted). In line with this, Fig. 7 illustrates that in the selected ROIs, the highest temperature drop $\left(\sim 3{ }^{\circ} \mathrm{C}\right)$ is achieved between 50 and $60 \mathrm{~g} \mathrm{~m}^{-2}$ and no further drop occurs beyond these values. This indicates that there is a moisture threshold causing a temperature limit, this possibly due to the attainment of maximum evaporative cooling. The data suggest that above this moisture threshold, variations in locals sweat content cannot be discriminated by temperature changes measured with IRT. In the currently adopted climatic conditions, this threshold corresponded to moisture content of approximately $50 \mathrm{~g} \mathrm{~m}^{-2}$, this causing a temperature drop limited to approximately $3{ }^{\circ} \mathrm{C}$.

Another factor to consider when studying the link between regional sweat retention and temperature drop in clothing is the uniformity of sweat/temperature distribution in each preselected garment region. Specifically, the temperature maps in Fig. $4 \mathrm{a}$ (front) and $4 \mathrm{~b}$ (back) show that changes in garment regional temperature do not occur uniformly within peripheral and inferior regions (e.g. front and back sleeves, mid back later, chest and abdomen lateral and lower ends). On the other hand, as the gravimetric method only allows an overall measurement of sweat retention in pre-defined regions, the sweat maps suggest a uniform distribution of the sweat absorbed in each region (Fig. 4a, b). This discrepancy could have affected the link between sweat retention and temperature change in those regions presenting a non-uniform sweat distribution. Further, the climatic condition adopted is another variable that can influence the relation between regional sweat retention and temperature drop. For instance, for the same garment saturation level, a higher temperature drop is expected in a dryer environment, as compared to the current conditions adopted (50\% Rh), thus improving the resolution and increasing the maximum temperature drop. Finally, using different fabrics can also affect the outcome. Given that absorptive capacity e.g. for synthetics is much lower than for the cotton used, it would be possible that for such fabrics, the water content limit of temperature drop would not be reached and thus a quantitative relation between temperature drop and water content may be observed after all. 
Table 4 Predictive equations of overall individual and average model as well as temporal models describing the statistical relation local sweat absorption (local temperature drop)

\begin{tabular}{lll}
\hline Model & Equation \\
\hline Overall individual & & ${ }^{10} \log \left(\mathrm{ABS}_{\text {Local }}\right)=0.615+1.014{ }^{10} \log \left(e^{\text {TempdropLocal }}\right)$ \\
Overall average & ${ }^{10} \log \left(\mathrm{ABS}_{\text {Local }}\right)=0.517+1.244{ }^{10} \log \left(e^{\text {TempdropLocal }}\right)$ \\
Temporal & $\left(\mathrm{ABS}_{\text {Local }}\right)=3.322 e^{0.9397 \text { TempdropLocal }}$ \\
& $15 \mathrm{~min}$ & $\left(\mathrm{ABS}_{\text {Local }}\right)=3.9442 e^{1.1368 \text { TempdropLocal }}$ \\
& $26 \mathrm{~min}$ & $\left(\mathrm{ABS}_{\text {Local }}\right)=3.6698 e^{1.2211 \text { TempdropLocal }}$ \\
& $35 \mathrm{~min}$ & $\left(\mathrm{ABS}_{\text {Local }}\right)=12.919 e^{0.8566 \text { TempdropLocal }}$ \\
\hline
\end{tabular}

As such, while the studied principle remains the same, different regression equations would need to be used if different climatic conditions or fabrics are applied.

When participants' average data of each ROI are used, rather than all individual data points, a better prediction model, as compared to the individual one, is obtained $\left(r^{2}=0.75\right.$, Table 3). As such, in some research settings, i.e. in studies involving within-subjects comparisons of different T-shirts, shorts or trousers, etcetera, the use of participants' average data could improve the predictive power of regional sweat content.

\section{Temporal models}

While in the overall individual model, which includes all individual data taken at the 10 different time points, regional temperature can only explain $52 \%$ of the variance in garment local sweat retention, temporal average models, including sweat and temperature data for separated exercise durations (Fig. 6), present a stronger predictive power $\left(r^{2}=0.75-88\right)$. Nevertheless, Fig. 6 shows that with the increase in exercise duration (highlighted in each separated model) and local sweat retention, the curve moves up and the slope becomes progressively steeper rather than

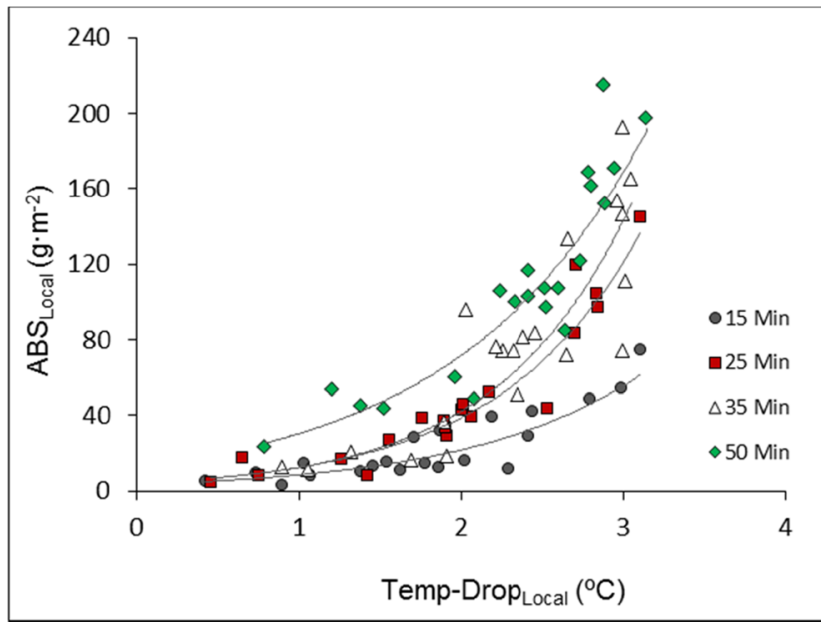

Fig. 6 Exponential relationships between local sweat absorption $\left(\mathrm{ABS}_{\text {Local }}\right)$ and local temperature drop $\left(\mathrm{TempDrop}_{\text {Local }}\right)$ at selected time points (15 min, $25 \mathrm{~min}, 35 \mathrm{~min}$ and $50 \mathrm{~min}$ ). Each model includes participants' average data for each ROI at a single time point $(21$ averaged ROIs $\times 1$ time point). The coefficient of determination $\left(R^{2}\right)$ of each model is reported in Table 3 developing towards the right side of the graph (increase in temperature drop). As indicated earlier, above a certain value, the increases in sweat retention, which occur as exercise time and sweat production progress, are not accompanied by concomitant increases in temperature drop. This again clearly shows that there is a temperature limit above which increases in sweat retention cannot be discriminated by using temperature drop values. Since sweat retention mainly changes as function of time, the overall model, including the temporal changes, is highly affected by this 'threshold effect' and shows a lower predictive power as compared to the single temporal models. However, such different temporal models at different exercise times would not be stable and would change with any change in condition. Thus, these are not practical in their application for quantitative sweat absorption determinations. As these temporal models do not allow accurate predictions of sweat retention between different exercise duration/intensity, IRT cannot be used to reliably quantitatively assess the development of sweat retention over time and across garment regions, unless sweat absorption remains very low. Nevertheless, the strong coefficient of determination of the temporal models indicates that IRT can very well be used to make qualitative interregional assessment of sweat retention, i.e. to define regions with high or low sweat content.

\section{Limitations}

The impact of body skin temperature on garment regional temperature was minimised by removing the garment from the body and allowing some time before performing the image acquisition. This time needs to be long enough in order to remove the effect of skin temperature variations and allow a steady-state garment temperature to be developed, but short enough to avoid sweat migration. In a pre-test, we observed that, in the first $3 \mathrm{~min}$ immediately after T-shirt removal, garment temperature dropped from 26 to $21.7^{\circ} \mathrm{C}$, leading to a difference of approximately $4{ }^{\circ} \mathrm{C}$. Natural wet bulb temperature (as indicator of the maximal temperature drop possible) in the climatic condition used was $20.8{ }^{\circ} \mathrm{C}$, therefore, approximately $6{ }^{\circ} \mathrm{C}$ difference from ambient temperature $\left(27{ }^{\circ} \mathrm{C}\right)$. However, although natural wet bulb temperature indicates that the limit for evaporative cooling was not fully achieved within the $3 \mathrm{~min}$, the data showed that the highest relative change of garment temperature $\left(1{ }^{\circ} \mathrm{C}\right)$ occurs during the first minute, 


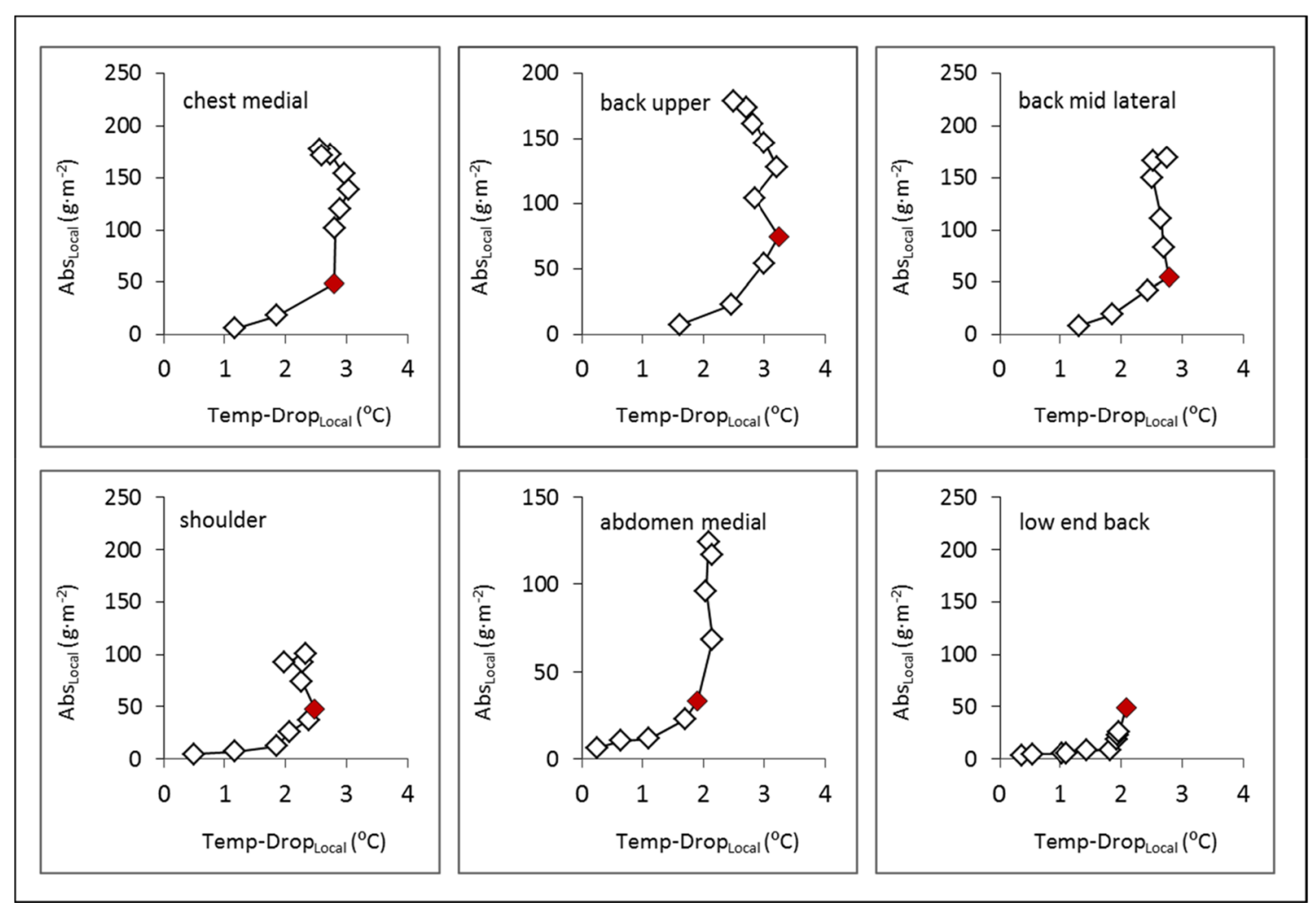

Fig. 7 Local sweat absorption $\left(\mathrm{ABS}_{\text {Local }}\right)$ plotted versus local temperature drop (TempDrop Local $\left._{1}\right)$ at each during duration $(5-50 \mathrm{~min})$ for selected regions of interest (ROI), i.e. chest medial, back upper,

immediately after fitting the garment on the stand. After $2 \mathrm{~min}$, this relative change accounts for $0.4{ }^{\circ} \mathrm{C}$, and after 2.5 to $8 \mathrm{~min}$, the relative change in garment temperature is very small $\left(0.1^{\circ} \mathrm{C}\right)$. Based on these data, a stabilisation period of 3 min was chosen, given that a longer stabilisation period between garment removal from the body and image acquisition could cause sweat migration across proximate regions.

An additional source of potential variation in the IR temperature measurement is the change in emission value when the T-shirt goes from dry (measured emission $(e)=0.91$ at 8 $12 \mu \mathrm{m})$ to wet $(e=0.97$ at $8-12 \mu \mathrm{m})$ (Hepokoski, personal communication). However, given the closeness of the T-shirt temperature to ambient (reflective) temperature, this variation would cause differences in the outcomes of less than $0.2{ }^{\circ} \mathrm{C}$.

Furthermore, a non-uniform contact between the T-shirt stand and garment could have affected the garment's temperature. Therefore, for future studies, a stand which has minimal contact area with the T-shirt, for instance a wire frame only in contact with the inner contour of the T-shirt yet allowing separation of the front and back garment's side, is proposed.

\section{Conclusions}

In this research, infrared thermography was used as a tool to indirectly quantify spatial and temporal variations in back mid latera, shoulder, abdomen medial and low end back. The red point indicates the highest TempDrop Local $_{\text {and }}$ related $\mathrm{ABS}_{\text {Local }}$ value

clothing sweat absorption, immediately after physical exercise of different duration/intensity. In order to minimise the impact of body skin temperature on garment local temperature, infrared image acquisition has to be performed after removal of the garment from the body, and after fitting it to a garment-shape stand. It can be concluded that, based on differences in local temperature drop (from dry state), IRT allows discrimination of higher and lower regional sweat retention, in a non-destructive way. Furthermore, IRT can be applied to make more precise qualitative assessments with regard to the level of sweat (temperature) distribution within a certain region, whereas the gravimetric method assumes uniformity of sweat retention in a pre-defined garment region. Nevertheless, despite these benefits, this study highlighted a number of limiting factors that preclude the use of IRT for quantitative estimations of spatial and temporal sweat retention in garments, when evaluations need to be performed within a relatively short time after exercise, with the main limitation being a moisture content threshold above which no further effect on temperature is observed. The latter may be less of an issue with synthetic garments with much lower absorptive capacity. Use of other wavelengths of electromagnetic radiation, e.g. at the absorption wavelength of water, may be another avenue to research in this context, in order to get to quantitative measurement. 


\section{Compliance with ethical standards}

Conflict of interest The authors declare that they have no conflict of interest.

Open Access This article is distributed under the terms of the Creative Commons Attribution 4.0 International License (http:// creativecommons.org/licenses/by/4.0/), which permits unrestricted use, distribution, and reproduction in any medium, provided you give appropriate credit to the original author(s) and the source, provide a link to the Creative Commons license, and indicate if changes were made.

\section{References}

Ammer K (2008) The Glamorgan protocol for recording and evaluation of thermal images of the human body. Thermology International 18: 125-129

Avdelidis NP, Moropoulou A, Theoulakis P (2003) Detection of water deposits and movement in porous materials by infrared imaging. Infrared Phys Technol 44:183-190

Baker LB, Reimel AJ, Sopeña BC, Barnes KA, Nuccio RP, de Chavez PJD, Stofan JR, Carter JM (2017) Trapped sweat in basketball uniforms and the effect on sweat loss estimates. Physiol Rep 5:e13463. https://doi.org/10.14814/phy2.13463

Balaras CA, Argiriou AA (2002) Infrared thermography for building diagnostics. Energ Buildings 34:171-183

British Standard European Norm International Organization for Standarization BS EN ISO 11092 (2014) Textiles-physiological effects - measurement of thermal and water-vapour resistance under steady-state conditions (sweating guarded-hotplate test)

British Standard European Norm International Organization for Standarization BS EN ISO 9237 (1995) Textiles-Determination of the permeability of fabrics to air

Costello JT, McInerney CD, Bleakley CM et al (2012) The use of thermal imaging in assessing skin temperature following cryotherapy: a review. J Therm Biol 37:103-110

Ferreira JJA, Mendonça LCS, Nunes LAO, Andrade Filho ACC, Rebelatto JR, Salvini TF (2008) Exercise-associated thermographic changes in young and elderly subjects. Ann Biomed Eng 36:1420 1427

Formenti D, Ludwig N, Trecroci A, Gargano M, Michielon G, Caumo A, Alberti G (2016) Dynamics of thermographic skin temperature response during squat exercise at two different speeds. J Therm Biol 59:58-63

Fournet D (2013) Skin temperature variations in the cold. PhD thesis Loughborough University

Fournet D, Ross L, Voelcker T, Redortier B, Havenith G (2013) Body mapping of thermoregulatory and perceptual responses of males and females running in the cold. J Therm Biol 38:339-344

Gerrett N, Ouzzahra Y, Redortier B, Voelcker T, Havenith G (2015) Female thermal sensitivity to hot and cold during rest and exercise. Physiol Behav 152:11-19
Hadžić V, Širok B, Malneršič A, Čoh M (2015) Can infrared thermography be used to monitor fatigue during exercise? A case study. J Sport Health Sci 8:89-92. https://doi.org/10.1016/J.JSHS.2015.08.002

IACT (2002) Thermography guidelines. Standards and protocols. http:// www.iact-org.org/professionals/thermog-guidelines.html

International Organization for Standardization ISO 9886:2004 (2004) Ergonomics-evaluation of thermal strain by physiological measurements

Mercer JB, Ring EFJ (2009) Fever screening and infrared thermal imaging: concerns and guidelines. Thermology International 19:67-69

Moreira DG, Costello JT, Brito CJ, Adamczyk JG, Ammer K, Bach AJE, Costa CMA, Eglin C, Fernandes AA, Fernández-Cuevas I, Ferreira JJA, Formenti D, Fournet D, Havenith G, Howell K, Jung A, Kenny GP, Kolosovas-Machuca ES, Maley MJ, Merla A, Pascoe DD, Priego Quesada JI, Schwartz RG, Seixas ARD, Selfe J, Vainer BG, Sillero-Quintana M (2017) Thermographic imaging in sports and exercise medicine: a Delphi study and consensus statement on the measurement of human skin temperature. J Therm Biol 69:155162. https://doi.org/10.1016/j.jtherbio.2017.07.006

Priego Quesada JI, Carpes FP, Bini RR, Salvador Palmer R, PérezSoriano P, Cibrián Ortiz de Anda RM (2015) Relationship between skin temperature and muscle activation during incremental cycle exercise. J Therm Biol 48:28-35

Raccuglia M, Hodder S, Havenith G (2016) Human wetness perception in relation to textile water absorption parameters under static skin contact. Text Res J 87:2449-2463

Raccuglia M, Heyde C, Lloyd A, et al (2017) T-shirt sweat absorption mapping. In: The 17th International Conference on Environmental Ergonomics. Kobe, Japan

Ring F, Ammer K (2000) Infrared imaging. Thermol Int 10:7-14

Robinson LJ, Law JM, Symonds ME, Budge H (2016) Brown adipose tissue activation as measured by infrared thermography by mild anticipatory psychological stress in lean healthy females. Exp Physiol 101:549-557

Selfe J, Alexander J, Costello JT, May K, Garratt N, Atkins S, Dillon S, Hurst H, Davison M, Przybyla D, Coley A, Bitcon M, Littler G, Richards J (2014) The effect of three different $\left(-135^{\circ} \mathrm{C}\right)$ whole body cryotherapy exposure durations on elite Rugby league players. PLoS One 9:e86420. https://doi.org/10.1371/journal.pone.0086420

Silva YA, Santos BH, Andrade PR, Santos HH, Moreira DG, SilleroQuintana M, Ferreira JJA (2017) Skin temperature changes after exercise and cold water immersion. Sport Sciences for Health 13: 195-202

Tang KPM, Kan CW, Fan JT (2014a) Assessing and predicting the subjective wetness sensation of textiles: subjective and objective evaluation. Text Res J 85:838-849

Tang KPM, Kan CW, Fan JT (2014b) Evaluation of water absorption and transport property of fabrics. Text Prog 46:1-132

Watmough DJ, Fowler PW, Oliver R (1970) The thermal scanning of a curved isothermal surface: implications for clinical thermography. Phys Med Biol 15:301 